\title{
Penetrative phototactic bioconvection
}

\author{
S. Ghorai ${ }^{1}$ and N.A. Hill ${ }^{2}$ \\ ${ }^{1}$ Department of Mathematics, Indian Institute of Technology, Kanpur 208016, India \\ ${ }^{2}$ Department of Mathematics, University of Glasgow, Glasgow G12 8QW, U.K.
}

\begin{abstract}
Using the generic model of Vincent and Hill [J. Fluid Mech, 327, 343 (1996)] for phototaxis in a suspension of swimming algae, we investigate two-dimensional phototactic bioconvection in a suspension confined between a rigid bottom boundary, and stressfree top and lateral boundaries. Phototaxis denotes swimming towards (positive) or away (negative) from light. The model of Vincent and Hill also incorporates the effect of shading where microorganisms close to the light source absorb and scatter light before it reaches those further away. The system is governed by the Navier-Stokes equations for an incompressible fluid coupled with a microorganism conservation equation. These equations are solved numerically using a conservative finite-difference scheme. Convection driven by phototactic microorganisms, which are slightly denser than water, has been investigated in a series of numerical experiments. The solutions show transition from steady state to periodic oscillations, and periodic oscillations to steady state to periodic oscillations again, as the governing parameters are varied. The mechanism driving the oscillatory solution just above the critical parameter values is explained.
\end{abstract}




\section{INTRODUCTION}

Bioconvection is the name given to spontaneous pattern formation in suspensions of microorganisms due to swimming of the microorganisms. ${ }^{1,2}$ In all cases the microorganisms are slightly denser than water and on average they swim upwards (although the reasons for up-swimming may be different for different species). Microorganisms respond to certain stimuli by tending to swim in particular directions. These responses are called taxes, examples being gravitaxis, gyrotaxis and phototaxis. Gravitaxis indicates swimming in the opposite sense to gravity and gyrotaxis is swimming directed by the balance between the torque due to gravity acting on a bottom-heavy cell and the torque due to viscous forces arising from local shear flows. Phototaxis denotes swimming towards or away from light. This paper is concerned with phototaxis only. Experimental observations have shown that bioconvection patterns in suspensions of single-celled algae are modified by illumination. ${ }^{3-5}$ Strong light destroys steady patterns in suspensions of microorganisms or prevents formation of patterns in well-stirred cultures. Light can also modify the shape or size of the pattern. The possible reasons for the changes in bioconvection pattern due to light intensity are the following. Firstly, the microorganisms are strongly phototactic. Most phototactic microorganisms are positively phototactic, i.e. they swim towards the light source, when the light intensity $I$ is below a critical value, $I_{c}$, and are negatively phototactic when $I>I_{c}{ }^{6}{ }^{6}$ Possible mechanisms by which the cells detect light and interpret the signal that they receive are discussed in Refs. 7-9. Thus cells tend to accumulate at optimal places in their environment where $I \approx I_{c}$. The second reason for pattern change arises due to shading. Turbidity in the water column causes $I$ to decrease with depth in the natural environment, but cells also absorb and to some extent scatter light, which decreases $I$ further. This latter effect is called self-shading and is the dominant effect in laboratory cultures. In this paper we have considered self-shading only. Self-shading determines the position in the suspension where $I=I_{c}$. If the volume fraction of cells in the suspension is small and the scattering of light by the cells in the suspension is weak, then the light intensity $I(x, y, z)$ received by an algal cell at position $(x, y, z)$ is given 
by the Lambert-Beer law: ${ }^{10,11}$

$$
I(x, y, z)=I_{s} \exp \left(-\alpha \int_{\gamma} n d s\right) .
$$

Here $\gamma$ is the straight line segment joining $(x, y, z)$ to the source of light intensity $I_{s}$, $\alpha$ is the extinction coefficient, and $n$ is the concentration of cells. All the effects of multiple scattering have been neglected, so an algal cell detects light that is reaching to it in a direct line from the source. When the suspension is uniformly illuminated from above, the mean swimming direction is given by

$$
<\boldsymbol{p}>=T(I) \hat{\boldsymbol{z}}
$$

where $\hat{\boldsymbol{z}}$ is the unit vector in the vertical direction. $T(I)$ is the phototaxis function such that

$$
T(I) \begin{cases}\geq 0 & \text { if } I \leq I_{c} \\ <0 & \text { if } I>I_{c} .\end{cases}
$$

$T(I)$ depends on the light intensity $I$ reaching the cell and the exact functional form of $T$ will depend on the species of microorganisms. The expression for $\langle\boldsymbol{p}\rangle$ in Eq. (2) is based on the assumption of purely phototactic cells which receive light from the source directly above. Thus any non-zero horizontal component in the mean swimming velocity, due to the nonuniform horizontal concentration distribution and balance between viscous and gravitational torques, has been neglected. The effects of the polarization and wavelength of light are not considered either. This simple model is a valid limiting case to consider to understand the complexities of phototactic bioconvection before proceeding to more complex models.

For a suspension of finite depth illuminated from above, there is a basic, horizontally uniform, state in which there is a balance between phototaxis coupled with suspension shading and diffusion due to the random component of the cell's swimming motions. This results in a horizontal concentrated layer of microorganisms (the sublayer), the position of which depends on the light intensity. Only the region below the sublayer is gravitationally unstable; the region above is stable. Therefore, if the whole fluid layer becomes unstable, the fluid motions in the unstable layer must penetrate the 
upper stable layer. This is an example of penetrative convection which occurs in a wide variety of convection problems. ${ }^{12}$

Given the need to photosynthesize, many motile algae are strongly phototactic and thus it is necessary to include phototaxis in realistic models of their behavior. Bioconvection in a suspension of phototactic algae was examined by Vincent and Hill. ${ }^{13}$ They performed a linear stability analysis of the basic equilibrium solution and found stationary and oscillatory modes of disturbance at the onset of instability. Note that the basic equilibrium solution derived by Vincent and Hill was wrong although the conclusions of the article are still valid qualitatively. The correct solution is given below in section IV B. There have been numerical simulations on bioconvection due to gravitaxis by Harashima et al. ${ }^{14}$ and due to gyrotaxis by Ghorai and Hill. ${ }^{15-18}$ Recently, Hopkins and Fauci ${ }^{19}$ simulated two-dimensional bioconvection using point particles rather than a continuum model and examined the general effects of a variety of different responses by the microorganisms, including gyrotaxis and chemotaxis, but they did not make specific comparisons with any particular biological system. No numerical experiments have been carried out on a suspension of phototactic microorganisms to date. Therefore we examine phototactic bioconvection in two dimensions using a continuum model.

We study two-dimensional phototactic bioconvection in a layer confined by rigid bottom, and stress-free top and lateral boundaries in the nonlinear regime. The mathematical formulation of the continuum model is described first followed by a brief description of the computational method. The code is validated by comparing the critical Rayleigh number computed using the present code with the linear stability results. This is followed by the numerical results.

\section{MATHEMATICAL FORMULATION}

The geometry considered consists of a two-dimensional rectangular region of width $L$ and height $H$ referred to Cartesian coordinates $(x, z)$ with the $z$-axis pointing vertically upwards. Thus the flow is confined to the $x z$-plane and independent of $y$. 


\section{A. Governing equations}

In common with the previous models of bioconvection, ${ }^{1}$ we assume a monodisperse cell population which can be modelled by a continuous distribution. The suspension is dilute so that the volume fraction of the cells is small and cell-cell interactions are negligible. Each cell has a volume $\vartheta$ and density $\rho+\Delta \rho$, where $\rho$ is the density of the water in which the cells swim and $\Delta \rho / \rho \ll 1$. $\boldsymbol{u}$ is the average velocity of all the material in a small volume $\delta V$ and $n$ is the cell concentration. Supposing that the suspension is incompressible and introducing stream function $\psi$ and vorticity $\zeta$, we get

$$
\boldsymbol{u}=(u, v, 0)=\left(\frac{\partial \psi}{\partial z},-\frac{\partial \psi}{\partial x}, 0\right), \quad \zeta=-\nabla^{2} \psi
$$

Neglecting all forces on the fluid except the cell's negative buoyancy, $n g \vartheta \Delta \rho$ per unit volume where $g$ is the acceleration due to gravity, the momentum equation under the Boussinesq approximation leads to the vorticity equation

$$
\frac{\partial \zeta}{\partial t}+\nabla \cdot(\zeta \boldsymbol{u})=\nu \nabla^{2} \zeta-\frac{\Delta \rho g \vartheta}{\rho} \frac{\partial n}{\partial x}
$$

Here $\nu$ is the kinematic viscosity of the suspension which is assumed to be that of the fluid.

The equation for cell conservation ${ }^{13}$ is

$$
\frac{\partial n}{\partial t}=-\nabla \cdot \boldsymbol{J}
$$

where $\boldsymbol{J}$ is the flux of cells. $\boldsymbol{J}$ can be written as

$$
\boldsymbol{J}=n \boldsymbol{u}+n W_{c}<\boldsymbol{p}>-D \nabla n .
$$

Here the first term is the flux due advection of the cells by the bulk fluid flow. The second term in Eq. (7) arises due to the average swimming of the cells: $W_{c}<\boldsymbol{p}>$ is the average swimming velocity relative to the fluid and $W_{c}$ is assumed to be constant. $<\boldsymbol{p}>$, given by Eq. (2), represents the average orientation of cells. The diffusive flux, which represents the random component of the cell locomotion, is given by the third term on the right hand side of Eq. (7). The diffusion coefficient $D$ is a constant which 
is based on the assumption that the diffusion tensor is a constant isotropic tensor. In principle, the diffusion tensor should be a function of light intensity and should be calculated from a swimming velocity autocorrelation function using generalised Taylor dispersion theory ${ }^{20,21}$ but no complete theory exists for all flows, and this simplification is needed to make realistic computational progress. Experience with bioconvection driven by gyrotaxis ${ }^{15-18}$ suggests that essential features of the flow will be captured. Assuming that the suspension is uniformly illuminated from above, Eq. (1) for light intensity becomes

$$
I(x, z)=I_{s} \exp \left(-\alpha \int_{z}^{H} n(x, z) \mathrm{d} z\right) .
$$

\section{B. Boundary conditions}

We impose a rigid, no-slip boundary condition on $z=0$ and require that both the normal velocity and tangential stress vanish on $z=H$. Also there is no flux of cells through $z=0, H$. Hence

$$
\left.\begin{array}{c}
\psi=0 \quad \text { and } \quad \boldsymbol{J} \cdot \hat{\boldsymbol{z}}=0 \quad \text { at } z=0, H \\
\frac{\partial \psi}{\partial z}=0 \quad \text { at } z=0, \quad \zeta=0 \quad \text { at } z=H .
\end{array}\right\}
$$

We require that both the normal velocity and tangential stress vanish on the side walls and no flux of cells through them, thus

$$
\psi=0, \quad \zeta=0, \quad \text { and } \quad \boldsymbol{J} \cdot \hat{\boldsymbol{x}}=0 \quad \text { at } \quad x=0, L .
$$

\section{Scaling of the equations}

Length is scaled on the depth $H$, velocity on $D / H$, time on the diffusive scale $H^{2} / D$, and the cell concentration on the mean cell concentration $\bar{n}$. In terms of the nondimensional variables, the bioconvection equations become

$$
\boldsymbol{u}=(u, v, 0)=\left(\frac{\partial \psi}{\partial z},-\frac{\partial \psi}{\partial x}, 0\right), \quad \zeta=-\nabla^{2} \psi
$$




$$
\frac{\partial \zeta}{\partial t}+\nabla \cdot(\zeta \boldsymbol{u})=S_{c} \nabla^{2} \zeta-S_{c} R \frac{\partial n}{\partial x}
$$

and

$$
\frac{\partial n}{\partial t}=-\nabla \cdot \boldsymbol{J}
$$

where the flux of cells is

$$
\boldsymbol{J}=n \boldsymbol{u}+n V_{c}<\boldsymbol{p}>-\nabla n
$$

Here $S_{c}=\nu / D$ is the Schmidt number, $V_{c}=W_{c} H / D$ is the scaled swimming speed and

$$
R=\frac{\bar{n} \vartheta \Delta \rho g H^{3}}{\rho \nu D}
$$

is the Rayleigh number. Also $\langle\boldsymbol{p}\rangle=T(I) \hat{\boldsymbol{z}}$ with $I$ given by

$$
I(x, z)=I_{s} \exp \left(-\kappa \int_{z}^{1} n(x, z) \mathrm{d} z\right)
$$

where $\kappa=\alpha \bar{n} H$ measures the strength of the absorption.

To estimate the various non-dimensional parameters, we assume that we are dealing with a purely phototactic microorganism, otherwise similar to Chlamydomonas. Typical values of these parameters are given in Table I based on estimates given by Kessler. ${ }^{22}$ The estimate for the extinction coefficient $\alpha$ is not available at present. However the non-dimensional parameter $\kappa$ is proportional to $\alpha$. Thus the effect of $\alpha$ can be studied by taking different values of $\kappa$.

Equations (11)-(14) have to be solved in the region $0 \leq x \leq \lambda, 0 \leq z \leq 1$, where $\lambda=L / H$ is the normalized width of the domain. These equations are subject to the boundary conditions

$$
\left.\begin{array}{c}
\psi=0 \quad \text { and } \quad \boldsymbol{J} \cdot \hat{\boldsymbol{z}}=0 \quad \text { at } z=0,1, \\
\frac{\partial \psi}{\partial z}=0 \quad \text { at } z=0, \quad \zeta=0 \quad \text { at } z=1,
\end{array}\right\}
$$

and

$$
\psi=0, \quad \zeta=0, \quad \text { and } \quad \boldsymbol{J} \cdot \hat{\boldsymbol{x}}=0 \quad \text { at } \quad x=0, \lambda .
$$

The boundary conditions (16) and (17) mean that the governing equations need only be solved over half of the full convection cell that extends from $x=0$ to $x=2 \lambda$; the 
solutions show mirror symmetry about the planes $x=0, x=\lambda$. Similar boundary conditions were used in numerical experiments on two-dimensional Rayleigh-Bénard convection by Moore and Weiss. ${ }^{23}$ These boundary conditions enforce no horizontal movement at $x=0$ and $x=\lambda$. Greater generality could be obtained by computing solutions in the full convection cell with periodic boundary conditions at $x=0$ and $x=2 \lambda$, but use of the boundary conditions (16) and (17) also allows us to use finer meshes at high Rayleigh numbers since the computations are being carried out on half of the solution domain.

The initial conditions are that of a uniform state together with a sinusoidal perturbation to a uniform concentration of cells of the form

$$
\psi=0, \quad \zeta=0, \quad n=1+\epsilon \cos (\pi x / \lambda)
$$

where $\epsilon=10^{-5}$. The perturbation is applied for computational convenience to ensure that one convection cell develops initially in the domain. Note that even if we do not apply a perturbation, rounding errors generate perturbations which initiate a single convection cell solution at an early stage, whenever the system is unstable.

\section{NUMERICAL PROCEDURE}

The governing Eqs. (11)-(14) are discretized using a conservative finite-difference scheme $^{24}$ on a staggered mesh with the stream function and vorticity stored on one set of nodes and the cell concentration stored on another set of nodes. The grid is chosen so that the concentration nodes lie in the interior only, whereas those of the stream function and vorticity lie in the interior and on the boundary of the domain. The advantage of the staggered mesh is that the no-cell flux boundary condition can be satisfied immediately when discretized without further approximation. We have used the well-known Woods' formula ${ }^{25}$ for the vorticity value on the boundary. This is obtained by expanding the stream function near the rigid surface using a three-term 
Taylor series expansion and the no-slip condition

$$
\zeta_{\mathrm{w}}=-\frac{\zeta_{\mathrm{nw}}}{2}-\frac{3 \psi_{\mathrm{nw}}}{(\Delta n)^{2}}
$$

where $\zeta_{\mathrm{nw}}, \psi_{\mathrm{nw}}$ are the values of $\psi, \zeta$ at the near-wall node (adjacent to the wall) and $\Delta n$ is the non-dimensional distance of the near-wall node from the wall.

An implicit scheme with Euler backward differencing in time and central differencing in space is used to discretize the governing equations. A line-by-line tridiagonal matrix algorithm with relaxation is used to solve the discretized equations. Some of the results were run with different numbers of grid points to check the grid independence of the solutions.

\section{LINEAR STABILITY OF THE BASIC EQUI- LIBRIUM STATE}

\section{A. Basic equilibrium solution}

Equations (11)-(14) possess a steady solution in which $\psi=\zeta=0$ and the concentration profile $n_{p}(z)$ is independent of $x$ and satisfies

$$
\frac{\mathrm{d} n_{p}}{\mathrm{~d} z}-V_{c} T(I) n_{p}=0 .
$$

Equation (20) is supplemented by the cell conservation relation

$$
\int_{0}^{1} n_{p} \mathrm{~d} z=1
$$

In terms of the new variable

$$
\omega=-\int_{z}^{1} n_{p} \mathrm{~d} z
$$

Eq. (20) becomes

$$
\frac{\mathrm{d}^{2} \omega}{\mathrm{d} z^{2}}-V_{c} T(I) \frac{\mathrm{d} \omega}{\mathrm{d} z}=0
$$

with boundary conditions

$$
\left.\begin{array}{ll}
\omega+1=0 & \text { at } z=0 \\
\omega=0 & \text { at } z=1
\end{array}\right\} .
$$


Here $I$ is given by

$$
I=I_{s} \exp (\kappa \omega)
$$

Equations (22) and (23) constitute a boundary value problem which is solved numerically using a shooting method.

Figure 1 shows two typical taxis functions of different critical intensities and the corresponding concentration profiles. The taxis functions were generated by superimposing the following sine functions.

$$
T(I)=0.8 \sin \left(\frac{3 \pi}{2} \chi(I)\right)-0.1 \sin \left(\frac{\pi}{2} \chi(I)\right),
$$

where $\chi(I)=I \exp (\beta(I-1))$. The value of critical intensity is related to the parameter $\beta$. When $I_{c}=I_{s}$, i.e. the critical intensity occurs at the upper surface, the cells accumulate at the top of the domain. This is similar to the gravitactic cells. As $I_{c}$ decreases, the maximum concentration decreases and the location of the maximum concentration shifts towards the mid-height of the domain. The maximum concentration is smallest when the maximum is located at the middle of the domain. As $I_{c}$ decreases further, the maximum concentration increases and shifts towards the bottom of the domain.

\section{B. Analytic equilibrium solution for weak absorption}

Vincent and Hill ${ }^{13}$ considered the "weak-absorption" case in which $0<\kappa \ll 1$ and $I$ is close to $I_{c}$ throughout the domain. Thus $T(I)$ is approximately a linear function of $I$,

$$
T(I)=-\Lambda\left(I-I_{c}\right)
$$

For comparison with the solutions in Ref. 13, we take the top and bottom boundaries at $z=0$ and $z=-1$ respectively. If the critical intensity $I_{c}$ occurs at position $z=-C$ $(0 \leq C \leq 1)$ for the vertically uniform concentration profile $n=1$, then

$$
T(I)=\Lambda I_{c} \kappa\left(\int_{z}^{0} n \mathrm{~d} z-C\right) .
$$

In this case the steady-state cell concentration satisfies ${ }^{13}$

$$
\frac{\mathrm{d} n_{p}}{\mathrm{~d} z}-d n_{p}\left[\int_{z}^{0} n_{p} \mathrm{~d} z-C\right]=0,
$$


subject to the cell conservation condition

$$
\int_{-1}^{0} n_{p} \mathrm{~d} z=1
$$

Here $d=V_{c} \Lambda I_{c} \kappa$ is a new constant. The unique solution of Eq. (25) can be obtained analytically as

$$
n_{p}(z)=\frac{\frac{K^{2}}{2 d}\left(\frac{K^{2}}{d^{2}}-C^{2}\right) \operatorname{sech}^{2}\left(\frac{K z}{2}\right)}{\left[\frac{K}{d}+C \tanh \left(\frac{K z}{2}\right)\right]^{2}}
$$

where $K$ is a constant determined (using Eq. (26)) from the equation

$$
\left(\frac{K^{2}}{d^{2}}+C-C^{2}\right) \tanh \left(\frac{K}{2}\right)-\frac{K}{d}=0
$$

The concentration profile in Eq. (27) is different to that of Vincent and Hill ${ }^{13}$ because the location of the maximum of the equilibrium concentration and thus the depth at which $I=I_{c}$ are not at $z=-C$ (as assumed in Ref. 13); they are both located above $z=-C$ if $0<C<1 / 2$ and below $z=-C$ if $1 / 2<C<1$. This can be understood as follows. Lack of symmetry in the concentration profile for $C \neq 1 / 2$, as it develops from the uniform state, leads to a difference in the diffusive flux above and below the line on which $I=I_{c}$. This causes the line on which $I=I_{c}$ to move to a different depth. The solutions in Ref. 13 are correct only for $C=0,1 / 2$ and 1 . For $C=0$ and 1 , the location of the line on which $I=I_{c}$ remains at the top and bottom of the domain respectively. When $C=1 / 2$, the symmetry in the concentration profile causes the line $I=I_{c}$ to stay at the middle of the domain.

\section{Code Validation}

To validate the bioconvection code, we now perform a linear stability analysis of the basic equilibrium solution. We introduce a small perturbation to the equilibrium solution $\psi=\zeta=0$ and $n=n_{p}(z)$ and the perturbed quantities are substituted into the governing equations and boundary conditions. Linearizing about the equilibrium state and resolving the perturbed quantities into normal modes produce linear stability equations. The linear stability equations are solved using a fourth-order finite-difference scheme, 
supplied by Dr. D.R. Moore ${ }^{26}$ that iterates using the Newton-Raphson-Kantorovich algorithm. We compute the critical Rayleigh number as a function of the wavelength using the present bioconvection code and compare the results with those of the linear stability analysis. The numerical solutions (using the present code) were obtained in a uniform mesh of sizes $\Delta x=0.025, \Delta y=0.025$ in the following way. First a solution for a single convection cell was established. This solution is one half of the periodic solution of wavelength twice the domain width. Then the value of the Rayleigh number was decreased by $5 \%$ and the new solution was allowed to settle to its steady state. The critical Rayleigh number was taken to be approximately the value at which the final steady state is first equal to the equilibrium state. The critical Rayleigh number computed from the bioconvection code lies within $\pm 1 \%$ of the value computed from the linear stability problem. Figure 2 shows the neutral curves for $V_{c}=10, I_{s}=0.5$ and $\kappa=1.0$ for two different critical intensities. The most unstable mode is stationary in both cases and the agreement between the linear stability analysis and the numerical results is satisfactory.

\section{RESULTS}

We have systematically investigated the effect of varying the Rayleigh number keeping the other parameters $S_{c}, I_{s}, I_{c}, V_{c}$ and $\kappa$ fixed. Due to large number of parameters, it is difficult to obtain a comprehensive picture across the whole parameter domain. Thus we take a discrete set of fixed parameter values to study their effect on the solution. The values of $S_{c}=20, I_{s}=0.8$ are kept fixed throughout. $I_{s}$ is the highest value of $I$ and the lowest value of I in our simulations is about 0.3 ; for this range of values of $I, T(I)$ is monotonically decreasing (see Fig. 1). We take $V_{c}=10,15,20$ as the representative parameter values for the swimming speed. For a given value of $V_{c}$ we take two values $\kappa=0.5,1.0$ of the absorption coefficient. Then for a given value of $\kappa$ we consider two values of critical intensity $I_{c}$ such that the location of the maximum static equilibrium concentration occurs around $z=3 / 4$ and $z=1 / 2$ respectively. The critical Rayleigh 
number $R_{c}$ and wavelength $\lambda_{c}$ are calculated for the given values of the parameters $V_{c}, \kappa$ and $I_{c}$ by solving the linear stability equations for the basic equilibrium solution given by Eqs. (22) and (23). The normalized width of the domain is set as $\lambda=\lambda_{c} / 2$ and the Rayleigh number is varied in the range $R_{c}<R<100 R_{c}$ to study penetrative phototactic bioconvection. The details of the computations are described below.

\section{A. $V_{c}=10$}

The representative values of $I_{c}$ and the corresponding static equilibrium concentration profiles are shown in Fig. 3. It shows that the concentration profile becomes steeper for the higher value of $\kappa$ when other parameter values are kept constant. Table II shows the critical Rayleigh numbers and the wavelengths for these values of $I_{c}$.

- Absorption coefficient $\kappa=0.5$. When $I_{c}=0.66$, the maximum of the equilibrium concentration occurs at around $z=3 / 4$. For $R=1.5 R_{c}$ we observe a steady state solution with a single convection cell with the higher cell concentration (plume) along the left wall (see Fig. 4(a)). This is an anti-clockwise solution and by symmetry there also exists a clockwise single convection solution with the plume located along the right wall. As $R$ is increased this single convection solution is no longer preferred. Instead we observe a two-cell convection solution with plume in the middle of the domain. This solution is shown for $R=5 R_{c}$ in Fig. 4(b) and it persists as $R$ is increased to $10 R_{c}$. As the value of $R$ is increased further, weak counter-rotating cells appear on top of the two main convection cells just described. One such solution is shown for $R=20 R_{c}$ (see Fig. $4(c))$ which is perfectly symmetric with respect to the vertical mid-line. These weak counter-rotating cells become stronger with increase in the value of $R$. This symmetric two-cell convection solution with counter-rotating cells on top persists for values of $R$ upto $40 R_{c}$. When $R$ reaches $50 R_{c}$, this solution loses its symmetry as seen in Fig. $4(\mathrm{~d})$, and this asymmetric solution persists as $R$ is increased to $60 R_{c}$. All the solutions for $R=1.5 R_{c}$ to $60 R_{c}$ are steady. When $R$ is increased further, the final state of the solution becomes periodic. One cycle of the oscillation for $R=70 R_{c}$ is shown in Fig. 5. Two, three and four convection cells appear in turn at the bottom of the domain 
in one oscillation cycle. The counter-rotating cells on the top disappear and reappear again during the cycle.

Next we consider $I_{c}=0.63$. In this case the maximum of the equilibrium concentration occurs near the mid height of the domain. As in the previous case, a single convection cell solution is observed for $R=1.5 R_{c}$ (see Fig. 6(a)). As the value of $R$ is increased to $5 R_{c}$, the preferred solution is two-cell convection with weak counter-rotating cells at the top layer. This two-cell convection solution with counter-rotating cells at the top persists as $R$ is increased to $30 R_{c}$. The counter-rotating cells at the top grow in size with the increase in the Rayleigh number. At $R=40 R_{c}$, we observe another set of counter-rotating cells appear on top the previous counter-rotating cells (see Fig. 6(c)). All the solutions from $R=5 R_{c}$ to $R=40 R_{c}$ possess symmetry with respect to the mid-vertical line. But this symmetry no longer exists for $R=50 R_{c}$ as shown in Fig. 6(d). At $R=60 R_{c}$ we observe a periodic solution that persists as $R$ is increased to $100 R_{c}$. The periodic solution is similar to the periodic solution observed for $I_{c}=0.66$ and $R=70 R_{c}$ (see the previous paragraph). During one cycle of the oscillation, two and three convection cells appear in turn at the bottom of the domain.

- Absorption coefficient $\kappa=1.0$. For $I_{c}=0.52$, all the solutions are steady for $R$ in the range from $1.5 R_{c}$ to $90 R_{c}$. For $R=1.5 R_{c}$ and $5 R_{c}$ we find a single convection cell solution. For $10 R_{c} \leq R \leq 70 R_{c}$, the steady state solutions consist of two-cell convection with counter-rotating cells near the top layer. The solutions for $10 R_{c} \leq R \leq 70 R_{c}$ are symmetric about the vertical mid-line. For $R=80 R_{c}$ and $90 R_{c}$ the steady solutions are not symmetric about the vertical mid-line. The solution becomes periodic when $R$ is increased to $100 R_{c}$.

For $I=0.50$, all the solutions for $R$ in the range from $1.5 R_{c}$ to $50 R_{c}$ are steady. A single convection cell solution is observed for $R=1.5 R_{c}$. For $R=5 R_{c}$ to $40 R_{c}$, the solutions consist of two-cell convection with counter-rotating cells near the upper surface. When $R$ becomes $50 R_{c}$ or higher the solution becomes periodic. 


\section{B. $V_{c}=15$ and $V_{c}=20$}

When $V_{c}=15$ and $\kappa=0.5$, the solutions are similar to those of $\kappa=0.5, V_{c}=10$. However some differences appear when $V_{c}=15, \kappa=1.0$. We start with $V_{c}=15, \kappa=1.0$ and $I_{c}=0.51$ for which the location of the maximum concentration occurs around $z=3 / 4$. When $R=1.5 R_{c}$ we observe a periodic solution instead of steady solution observed in the previous cases.

The evolution of central concentration, $n_{\text {mid }}=n(3 / 4,1 / 2)$, at the midpoint of the domain is shown in Fig. 7(a). The equilibrium solution becomes unstable around $t=3$. After some transient oscillations, the solution becomes periodic. The fluctuation of $n_{\text {mid }}$ in $6 \leq t \leq 10$ (see Fig. $7(\mathrm{a})$ ) corresponds to the periodic solution. The reason for observing this periodic solution is that the most unstable mode at the critical Rayleigh number is overstable, i.e. oscillatory (see Fig. 7(b)). The oscillatory mode at the critical state persists at $R=1.5 R_{c}$, and in Fig. 8 we show one cycle of oscillation of this solution.

The mechanism of the oscillations is explained as follows. If the Rayleigh number is near the threshold, the concentration contours will be horizontal with the maximum concentration located near $z=3 / 4$. Thus the region above the maximum concentration level will be stable and the region below will be unstable. Since $R=1.5 R_{c}$ is above the threshold value, the convection in the unstable region penetrates the upper stable region. Figure 8(a) shows a clockwise convection cell extending throughout the domain. As a result of clockwise convection the concentration in the right half of the domain is higher than that in the left half of the domain. The position of greatest concentration shifts up in the right half and vice versa. The location of the maximum concentration in the right and left halves changes accordingly. Thus in the unstable region of the right half domain the convection and vertical cell flux oppose each other. The convection acts to bring cells downward whereas the vertical cell flux pushes the cells upward. As a result the strength of the convection decreases. Meanwhile in the left half of the domain, the cells accumulate due to downward movement of the cells and cells carried by convection from the right half. The accumulated cells in the left half generate a anti- 
clockwise convection cell in the bottom left corner and the reduced convection strength in the right half no longer penetrates the whole layer. The clockwise convection cell moves to the top right corner and it reinforces the left anti-clockwise convection cell. Thus the left half convection cell grow in magnitude and ultimately fills the whole domain. The same cycle is again repeated in the left half of the domain and the periodic solution results.

The results for some other values of $R$ in this case also are different from the previous cases. Here for $R=5 R_{c}$ the solution is the two-cell convection and when $R=10 R_{c}$ the preferred solution consists of two-cell convection with counter-rotating cells at the top. A three-cell convection solution with counter-rotating cells at the top is observed for $R=20 R_{c}$ and $R=40 R_{c}$. When $R$ equals $50 R_{c}$, the preferred solution consists of four-cell convection with counter-rotating cells at the top. The last solution persists when $R$ becomes $100 R_{c}$. Also all the solutions mentioned in this paragraph are steady. In the previous cases, the preferred solutions at higher Rayleigh number are either steady two-cell convection with counter-rotating cells or periodic solutions. Here we see that the number of convection cells increases from two to four and all of them are steady. Figure 9 shows representative solutions in this case.

When $V_{c}=20, \kappa=0.5$ and $I_{c}=0.64$, the location of the maximum concentration occurs around $z=3 / 4$. The solution at $R=1.5 R_{c}$ is periodic. This is due to the overstability at the critical Rayleigh number and is similar to the case when $V_{c}=$ $15, \kappa=1.0$. The other solutions are similar to those when $V_{c}=15$.

\section{SUMMARY OF RESULTS}

All the results presented in the previous section are presented in Table III below, and the following notation is used. The notations $m$ and $m(s)$ represent $m$-cell convection and symmetric (with respect to mid vertical line) $m$-cell convection solutions respectively. The notations $m_{m}(s)$ and $m_{m}(n s)$ are used to represent symmetric $m$-cell convection with counter-rotating cells at the top and asymmetric $m$-cell convection 
with counter-rotating cells at the top. Finally $p$ stands for periodic solution. $I_{c 1}$ and $I_{c 2}$ denote the critical light intensities such that the maximum of the static equilibrium concentration located near $z=3 / 4$ and $z=1 / 2$ respectively. The critical wavelengths $\lambda_{c}$ for each set of parameter values are also shown in the last line of Table III.

\section{CONCLUSIONS}

A conservative finite difference method was applied to solve the phototactic bioconvection problem in two dimensions. The bioconvection model is based on the continuum model of Vincent and Hill ${ }^{13}$ which takes in to account the self-shading effect of the microorganisms. The results are presented for a set of values of swimming speed and absorption coefficient when the Rayleigh number is varied from $1.5 R_{c}$ to $100 R_{c}$. At low Rayleigh number, the solutions are steady except when the critical state is overstable. In that case the solution is oscillatory at $R=1.5 R_{c}$ and a simple mechanism for the oscillatory behaviour was presented in section V B. Usually the overstability occurs when the static equilibrium concentration has maximum near three-quarters of the height of the domain. The normalized width of the domain is chosen to be half of the wavelength at the corresponding critical number. Thus only one-cell convection is observed when the Rayleigh number is near critical value, i.e. $R=1.5 R_{c}$. At higher Rayleigh numbers, the single convection cell solution is not preferred; instead we observe multiple convection cells with counter-rotating cells at the top. In all of the simulation with lower absorption coefficient $(\kappa=0.5)$, the final state of the solution is periodic. In some cases with higher swimming speed and absorption coefficient, the final state might be steady. In that case usually the number of convection cells increases with an increase in the value of the Rayleigh number.

The experimental study of phototactic bioconvection presents some difficulties and, to date, there are no quantitative results. For comparison with our study, it will be necessary to identify a suitable species of micro-organisms that are predominantly phototactic. Most species of algae are gyrotactic or gravitactic, in addition to being 
phototactic (Hill and Häder, ${ }^{27}$ Kessler et $a l .{ }^{28}$ ). Experiments to determine taxis functions, extinction and diffusion coefficients etc. will also be required. We await with interest experiments on the fascinating phenomena revealed by these simulations.

\section{ACKNOWLEDGMENTS}

S.G. is grateful to Department of Science and Technology (India) for a BOYSCAST fellowship held while part of this research was being carried out at the Department of Mathematics, University of Glasgow, UK.

\section{References}

[1] T.J. Pedley and J.O. Kessler, "Hydrodynamic phenomena in suspensions of swimming microorganisms," Ann. Rev. Fluid Mech. 24, 313 (1992).

[2] N.A. Hill and T.J. Pedley, "Bioconvection," Fluid Dyn. Res. (Accepted) (2005).

[3] H. Wager, "On the effect of gravity upon the movements and aggregation of Euglena viridis. Ehrb., and other microorganisms," Phil. Trans. R. Soc. London, Ser. B 201, 333 (1911).

[4] J.O. Kessler, "Co-operative and concentrative phenomena of swimming microorganisms," Contemp. Phys. 26, 147 (1985).

[5] R.V. Vincent, "Mathematical modelling of phototaxis in motile microorganisms," Ph.D. thesis, University of Leeds, 1995.

[6] D.-P. Häder, "Polarotaxis, gravitaxis and vertical phototaxis in the green flagellate, Euglena gracilis," Arch. Microbiol. 147, 179 (1987).

[7] K.W. Foster and R.D. Smyth, "Light antennas in phototactic algae," Microbiol. Rev. 44, 572 (1980).

[8] N.A. Hill and R.V. Vincent, "A simple model and strategies for orientation in phototactic micro-organisms," J. Theor. Biol. 163, 223 (1993). 
[9] N.A. Hill and L.A. Plumpton, "Control strategies for the polarotactic orientation of the micro-organism Euglena gracilis," J. Theor. Biol. 203, 357 (2000).

[10] G. Herdan, Small Particle Statistics, 2nd ed. (Butterworth \& Co. London, 1960).

[11] L.N.M. Duysens, "The flattening of the absorption spectrum of suspensions, as compared to that of solutions," Biochim. Biophys. Acta 19, 1, (1956).

[12] B. Straughan, Mathematical aspects of penetrative convection, (Longman Scientific \& Technical, 1993).

[13] R.V. Vincent and N.A. Hill, "Bioconvection in a suspension of phototactic algae," J. Fluid Mech. 327, 343 (1996).

[14] A. Harashima, M. Watanabe, and I. Fujishiro, "Evolution of Bioconvection Patterns in a Culture of Motile Flagellates," Phys. Fluids 31, 764 (1988).

[15] S. Ghorai and N.A. Hill, "Development and stability of gyrotactic plumes in bioconvection," J. Fluid Mech. 400, 1 (1999).

[16] S. Ghorai and N.A. Hill, "Periodic arrays of gyrotactic plumes in bioconvection," Phys. Fluids 12, 5 (2000).

[17] S. Ghorai and N.A. Hill, "Wavelengths of gyrotactic plumes in bioconvection," Bull. Math. Biol. 62, 429 (2000).

[18] S. Ghorai and N.A. Hill, "Axisymmetric bioconvection in a cylinder," J. Theor. Biol. 219, 137 (2002).

[19] M.M. Hopkins and L.J. Fauci, "A computational model of the collective fluid dynamics of motile microorganisms," J. Fluid Mech. 455, 149 (2002).

[20] N.A. Hill and M.A. Bees, "Taylor dispersion of gyrotactic swimming microorganisms in a linear shear flow," Phys. Fluids 14, 2598 (2002).

[21] A. Manela and I. Frankel "Generalized Taylor dispersion in suspensions of gyrotactic swimming micro-organisms," J. Fluid Mech. 490, 99 (2003). 
[22] J.O. Kessler, "Individual and collective fluid dynamics of swimming cells," J. Fluid Mech. 173, 191 (1986).

[23] D.R. Moore and N.O. Weiss, "Two-dimensional Rayleigh-Bénard convection," J. Fluid Mech. 58, 289 (1973).

[24] S. Ghorai, "Bioconvection and plumes," Ph.D. thesis, University of Leeds, 1997.

[25] L.C. Woods, "A note on the numerical solution of fourth order differential equations," Aeronaut. Quart. 5, 176, (1954).

[26] J.R. Cash and D.R. Moore, "A high order method for the numerical solution of two-point boundary value problems," BIT 20, 44 (1980).

[27] N.A. Hill and D.-P. Häder, "A biased random walk model for the trajectories of swimming micro-organisms," J. theor. Biol. 186, 503 (1997).

[28] J.O. Kessler, N.A. Hill, and D.-P. Häder, "Orientation of swimming flagellates by simultaneously acting external factors," J. Phycology. 28, 816 (1992). 


\begin{tabular}{lll}
\hline Cell radius & $a$ & $10^{-3} \mathrm{~cm}$ \\
Cell volume & $\vartheta$ & $5 \times 10^{-10} \mathrm{~cm}^{3}$ \\
Cell density ratio & $\delta \rho / \rho$ & $5 \times 10^{-2}$ \\
Cell diffusivity & $D$ & $5 \times 10^{-4} \mathrm{~cm}^{2} \mathrm{~s}^{-1}$ \\
Swimming speed & $W_{c}$ & $10^{-2} \mathrm{~cm} \mathrm{~s}^{-1}$ \\
Mean concentration & $\bar{n}$ & $10^{6} \mathrm{~cm}^{-3}$ \\
Kinematic viscosity & $\nu$ & $10^{-2} \mathrm{~cm}^{2} \mathrm{~s}^{-1}$ \\
\hline
\end{tabular}

Table I: Estimates of typical parameters for a suspension of Chlamydomonas. 
(a)

\begin{tabular}{ccc}
\hline$I_{c}$ & $R_{c}$ & $\lambda_{c}$ \\
\hline 0.66 & 317 & 2.88 \\
0.63 & 1159 & 2.0 \\
\hline
\end{tabular}

(b)

\begin{tabular}{ccc}
$I_{c}$ & $R_{c}$ & $\lambda_{c}$ \\
\hline 0.52 & 391 & 2.0 \\
0.50 & 639 & 2.0 \\
\hline
\end{tabular}

Table II: Critical Rayleigh numbers and critical wavelengths at the onset of linear instability for $V_{s}=10, I_{s}=0.8$ : (a) $\kappa=0.5$, (b) $\kappa=1.0$. 


\begin{tabular}{|c|c|c|c|c|c|c|c|c|c|c|c|c|}
\hline \multirow{3}{*}{$\frac{R}{R_{c}}$} & \multicolumn{4}{|c|}{$V_{c}=10$} & \multicolumn{4}{|c|}{$V_{c}=15$} & \multicolumn{4}{|c|}{$V_{c}=20$} \\
\hline & \multicolumn{2}{|c|}{$\kappa=0.5$} & \multicolumn{2}{|c|}{$\kappa=1.0$} & \multicolumn{2}{|c|}{$\kappa=0.5$} & \multicolumn{2}{|c|}{$\kappa=1.0$} & \multicolumn{2}{|c|}{$\kappa=0.5$} & \multicolumn{2}{|c|}{$\kappa=1.0$} \\
\hline & $I_{c 1}$ & $I_{c 2}$ & $I_{c 1}$ & $I_{c 2}$ & $I_{c 1}$ & $I_{c 2}$ & $I_{c 1}$ & $I_{c 2}$ & $I_{c 1}$ & $I_{c 2}$ & $I_{c 1}$ & $I_{c 2}$ \\
\hline 1.5 & 1 & 1 & 1 & 1 & 1 & 1 & $p$ & 1 & $p$ & 1 & $p$ & 1 \\
\hline 5 & $2(s)$ & $2_{2}(s)$ & $"$ & $2_{2}(s)$ & $2(s)$ & $2_{2}(s)$ & $2(s)$ & $2_{2}(s)$ & $2(s)$ & $2_{2}(s)$ & $2(s)$ & $2_{2}(s)$ \\
\hline 10 & $"$ & $"$ & $2_{2}(s)$ & $"$ & $2_{2}(s)$ & $"$ & $2_{2}(s)$ & $"$ & $2_{2}(s)$ & $"$ & $3_{3}(s)$ & $"$ \\
\hline 20 & $2_{2}(s)$ & $"$ & $"$ & $"$ & $2_{2}(n s)$ & $"$ & $3_{3}(s)$ & $"$ & $3_{3}(s)$ & $"$ & $"$ & $"$ \\
\hline 30 & $"$ & $"$ & $"$ & $"$ & $p$ & $"$ & $"$ & $2_{2}(n s)$ & $"$ & $"$ & $"$ & $3_{3}(s)$ \\
\hline 40 & $"$ & $2_{2_{2}}(s)$ & $"$ & $"$ & $"$ & $p$ & $"$ & $p$ & $"$ & $p$ & $4_{4}(s)$ & $"$ \\
\hline 50 & $2_{2}(n s)$ & $2_{2_{2}}(n s)$ & $"$ & $p$ & $"$ & $"$ & $4_{4}(s)$ & $"$ & $"$ & $"$ & $4_{4}(s)$ & $"$ \\
\hline 60 & $"$ & $p$ & $"$ & $"$ & $"$ & $"$ & $"$ & $"$ & $p$ & $"$ & $5_{5}(s)$ & $"$ \\
\hline 70 & $p$ & $"$ & $"$ & $"$ & $"$ & $"$ & $"$ & $"$ & $"$ & $"$ & $4_{4}(s)$ & $"$ \\
\hline 80 & $"$ & $"$ & $2_{2}(n s)$ & $"$ & $"$ & $"$ & $"$ & $"$ & $"$ & $"$ & $"$ & $4_{4}(s)$ \\
\hline 90 & $"$ & $"$ & $"$ & $"$ & $"$ & $"$ & $"$ & $"$ & $"$ & $"$ & $5_{5}(s)$ & $"$ \\
\hline 100 & $"$ & $"$ & $p$ & $"$ & $"$ & $"$ & $"$ & $"$ & $"$ & $"$ & $6_{6}(s)$ & $"$ \\
\hline$\lambda_{c}$ & 2.88 & 2.0 & 2.0 & 2.0 & 2.8 & 1.94 & 3.0 & 1.86 & 2.9 & 1.86 & 3.2 & 1.8 \\
\hline
\end{tabular}

Table III: Summary of the numerical results. See the text for the explanation of the notations. The critical wavelengths are shown in the last line of the table. 


\section{Figure captions}

Fig. 1: (a) Typical taxis functions, and (b) the corresponding equilibrium concentration profile for $V_{c}=10, I_{s}=0.5, \kappa=1.0$. The taxis functions are generated by superimposing sine functions (see Eq. 24).

Fig. 2: Neutral curve (solid line for the stationary mode and dashed line for the oscillatory mode) at the onset of bioconvection for the system whose equilibrium solution is plotted in Fig. 1. The solutions obtained from the bioconvection code are marked on the lines using solid squares.

Fig. 3: Basic equilibrium concentration profiles for $V_{c}=10, I_{s}=0.8$ : (a) $\kappa=0.5$, (b) $\kappa=1.0$. The concentration profile becomes steeper for higher value of $\kappa$.

Fig. 4: Streamlines of steady solutions for $V_{c}=10, \kappa=0.5$ and $I_{c}=0.66$. The peak of the basic equilibrium concentration profile for these parameters is near the three-quarter height of the domain (see Fig. 3(a)).

Fig. 5: One cycle of the oscillation for $V_{c}=10, \kappa=0.5, I_{c}=0.66$ and $R=70 R_{c}$. The basic equilibrium concentration profile is shown in Fig. 3(a). The instantaneous streamlines are plotted at equal intervals of time and the period is approximately 0.17 in non-dimensional units.

Fig. 6: Streamlines of steady solutions for $V_{c}=10, \kappa=0.5$ and $I_{c}=0.63$. The peak of the basic equilibrium concentration profile for these parameters is near the mid-height of the domain (see Fig. 3(a)). New counter-rotating cells appear with increase in the value of $R$ prior to the periodic state.

Fig. 7: (a) Variation of the central concentration, $n_{\text {mid }}=n(3 / 4,1 / 2)$, with time for $R=1.5 R_{c}$ and (b) the corresponding neutral curve (solid line for the stationary mode and dotted line for the oscillatory mode) at the onset of bioconvection. The other parameter values are $V_{c}=15, \kappa=1.0$ and $I_{c}=0.51$. 
Fig. 8: One cycle of the oscillation for $V_{c}=15, \kappa=1.0, I_{c}=0.51$ and $R=1.5 R_{c}$. The peak of the basic equilibrium concentration profile for these parameters develops around three-quarter height of the domain. The instantaneous streamlines and concentration are plotted using solid and dotted lines respectively. The values on the concentration contours increase as we move towards the inner contours.

Fig. 9: Streamlines of steady solutions for $V_{c}=15, \kappa=1.0$ and $I_{c}=0.51$. The peak of the basic equilibrium concentration profile for these parameters is near the threequarter height of the domain. The number of convection cells increases with $R$ in the range from $R=1.5 R_{c}$ to $R=50 R_{c}$ and then remains constant as $R$ is increased to $100 R_{c}$ 
(a)

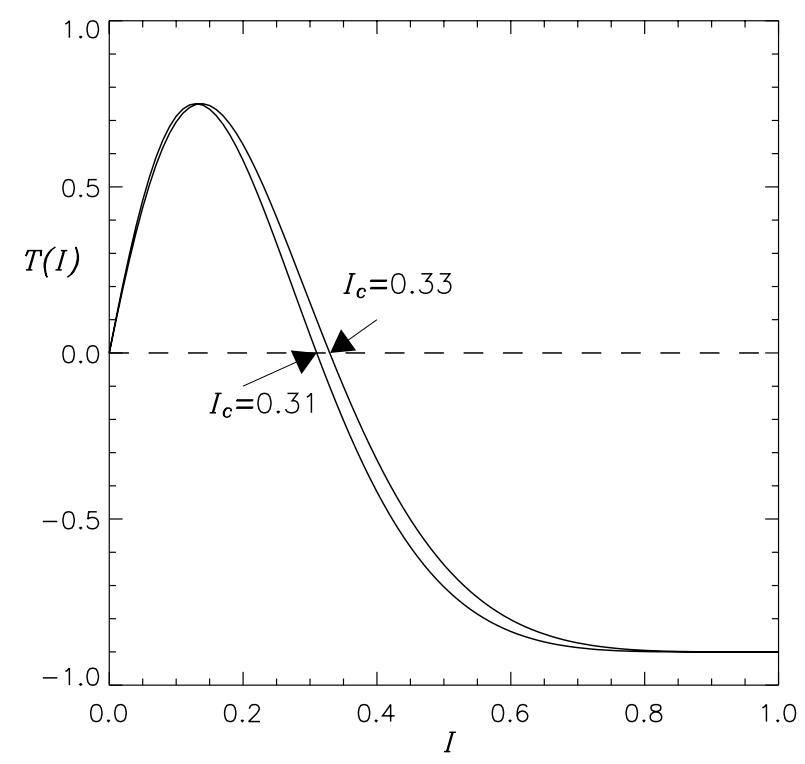

(b)

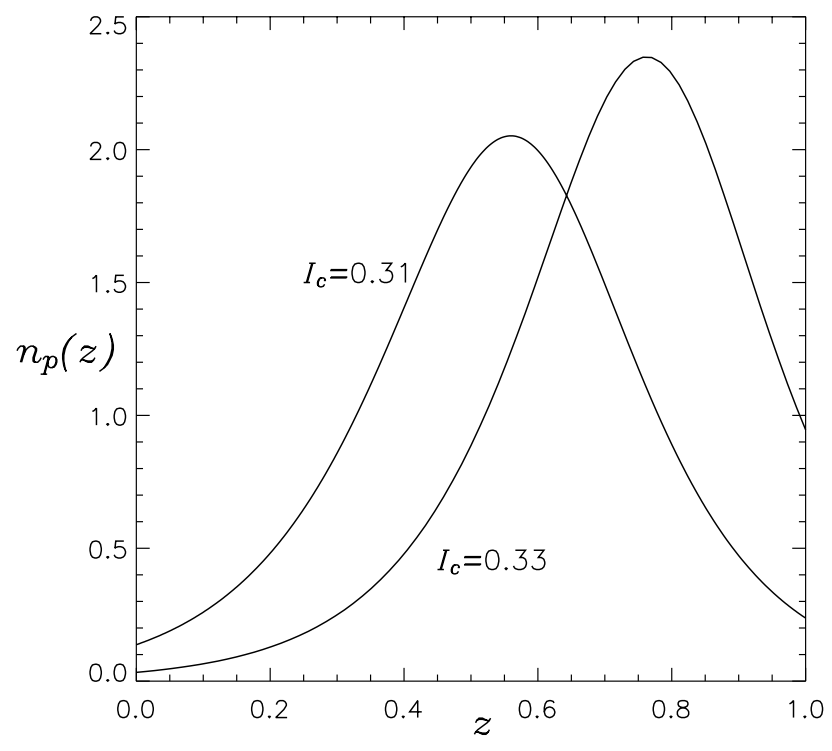

Figure 1: S Ghorai, Physics of Fluids 


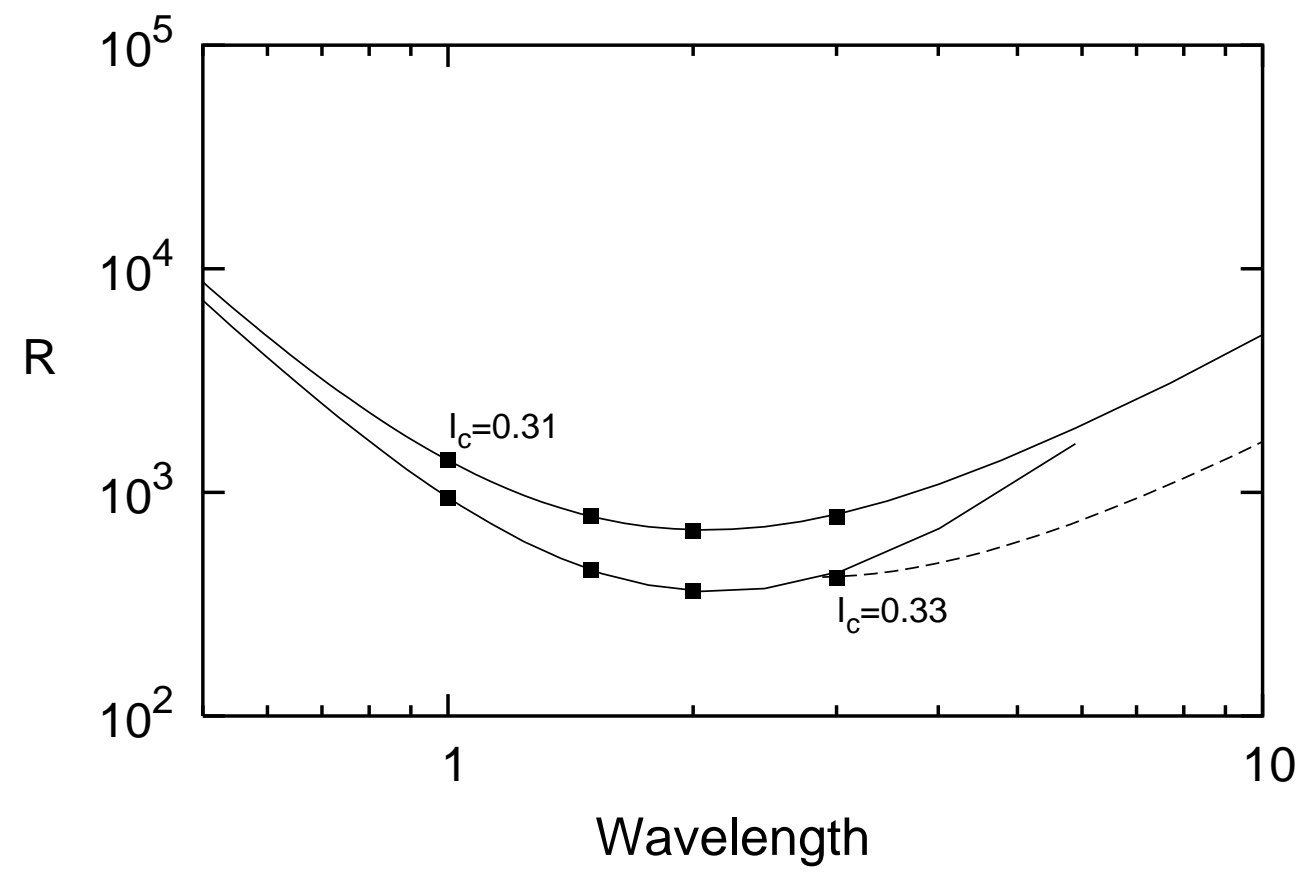

Figure 2: S Ghorai, Physics of Fluids 
(a)

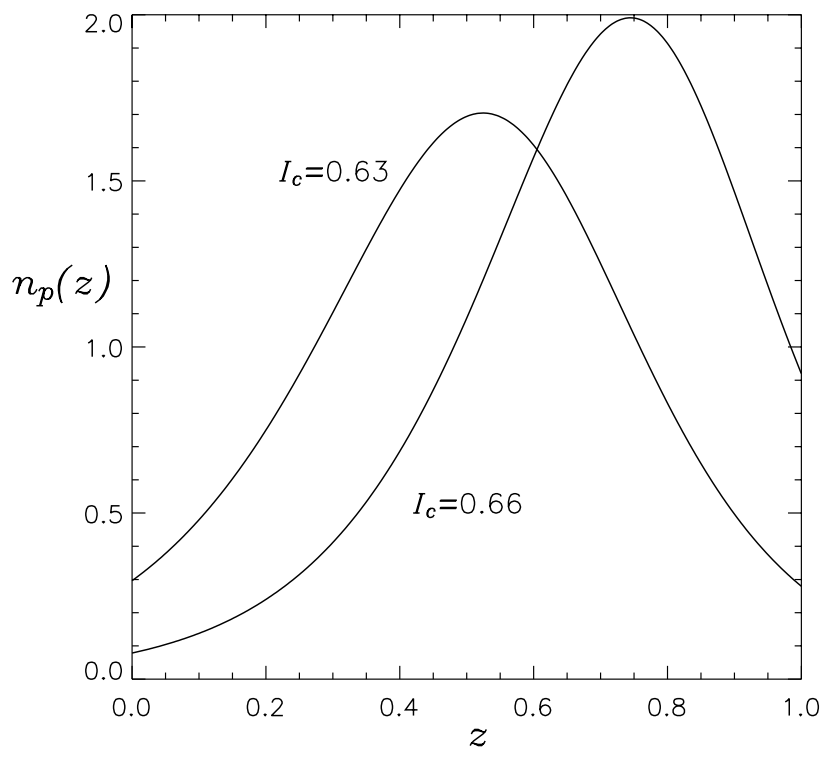

(b)

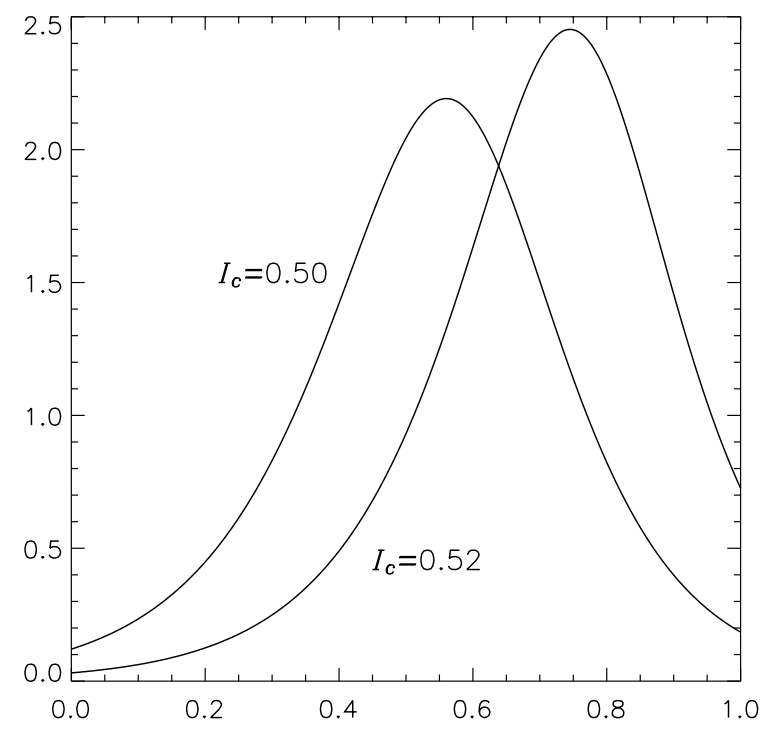

Figure 3: S Ghorai, Physics of Fluids 
(a) $R=1.5 R_{c}$

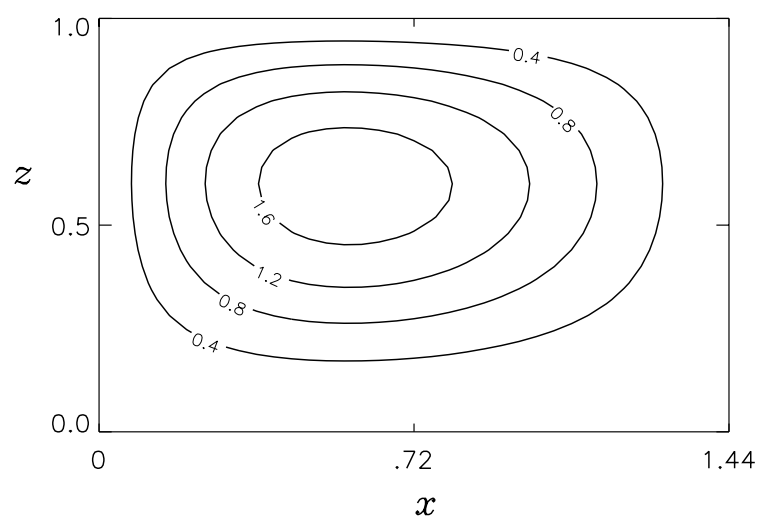

(c) $R=20 R_{c}$

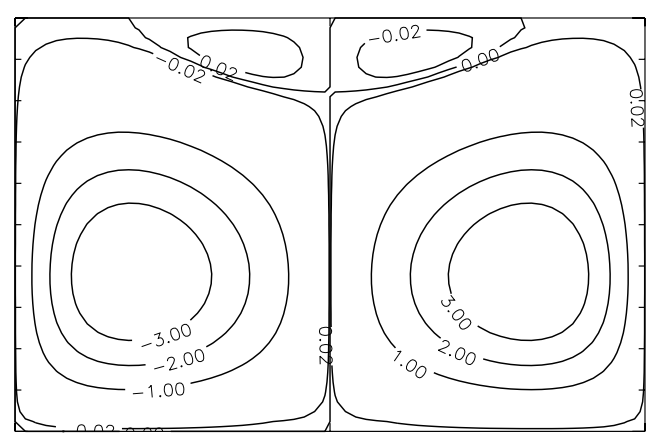

(b) $R=5 R_{c}$

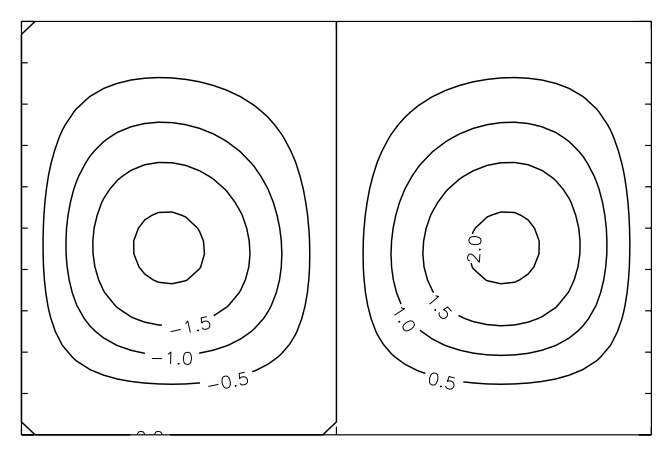

(d) $R=50 R_{c}$

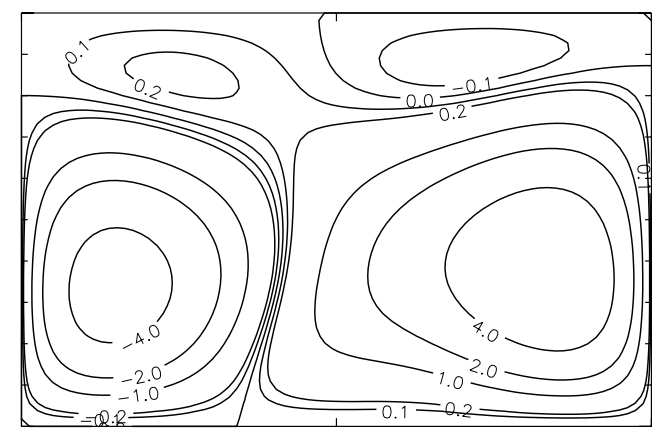

Figure 4: S Ghorai, Physics of Fluids 
(a)

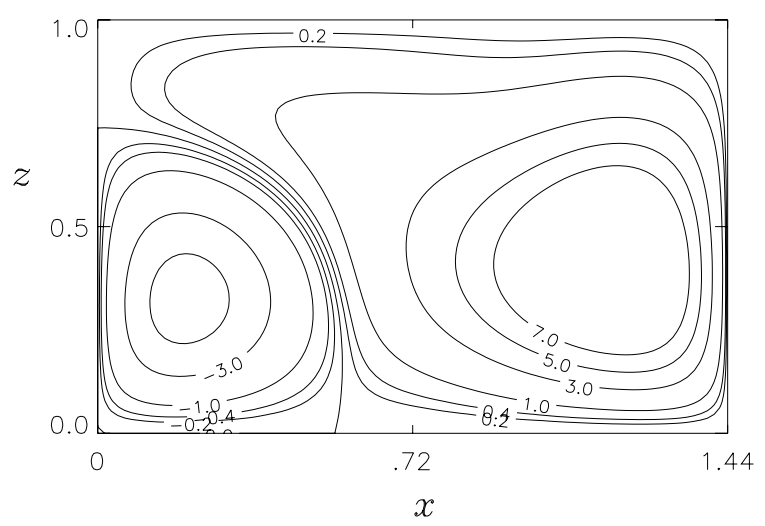

(c)

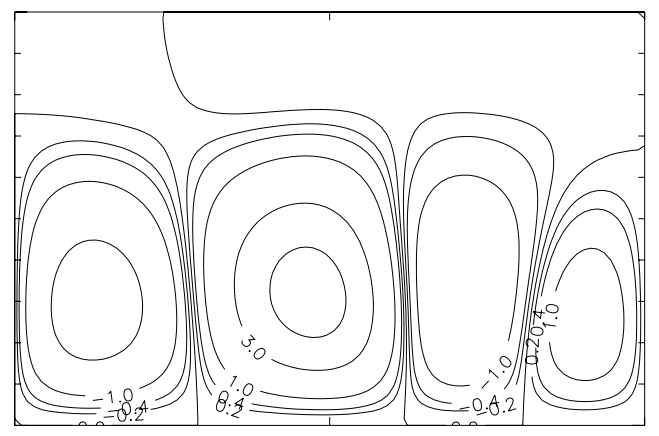

(e)

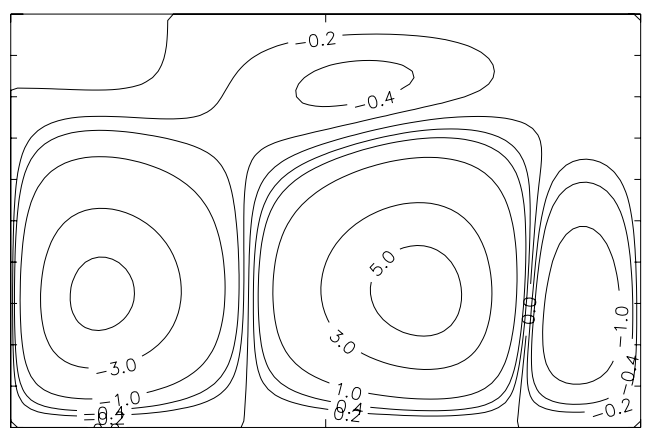

(b)

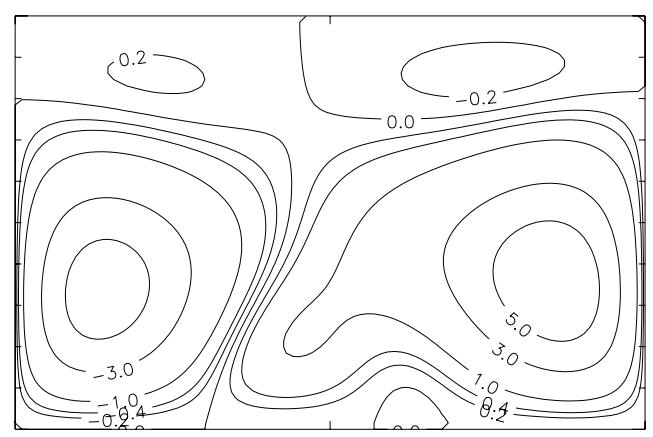

(d)

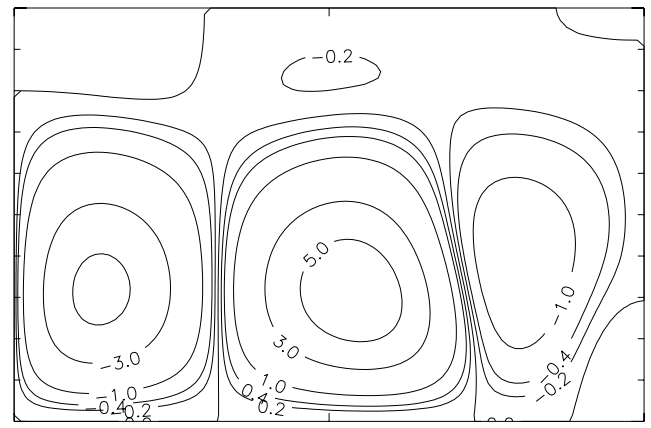

(f)

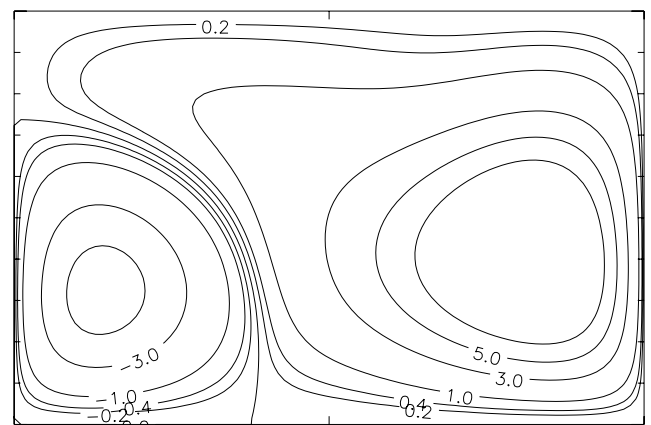

Figure 5: S Ghorai, Physics of Fluids 
(a) $R=1.5 R_{c}$

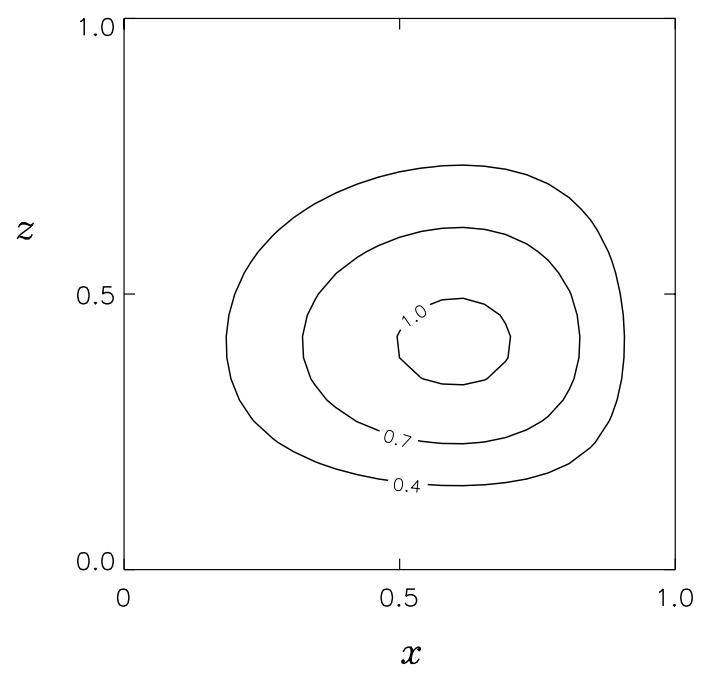

(c) $R=40 R_{c}$

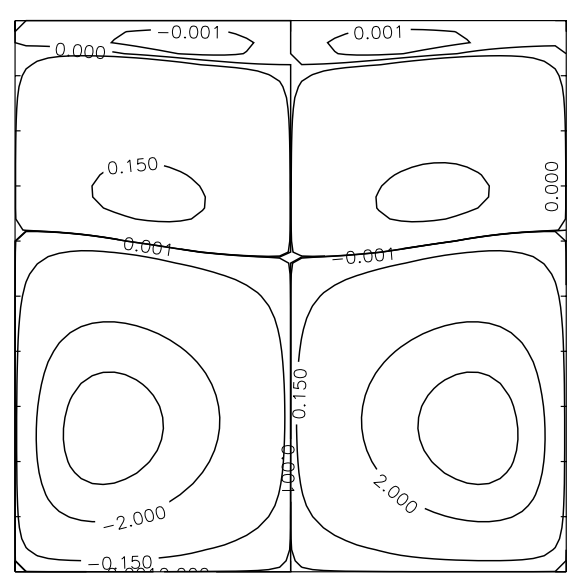

(b) $R=5 R_{c}$

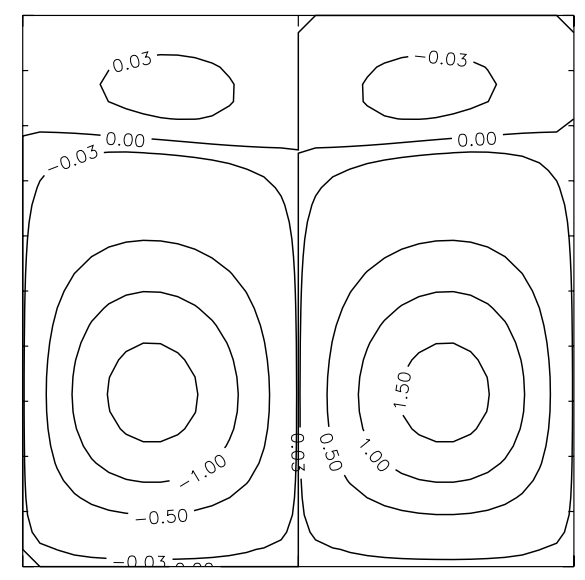

(d) $R=50 R_{c}$

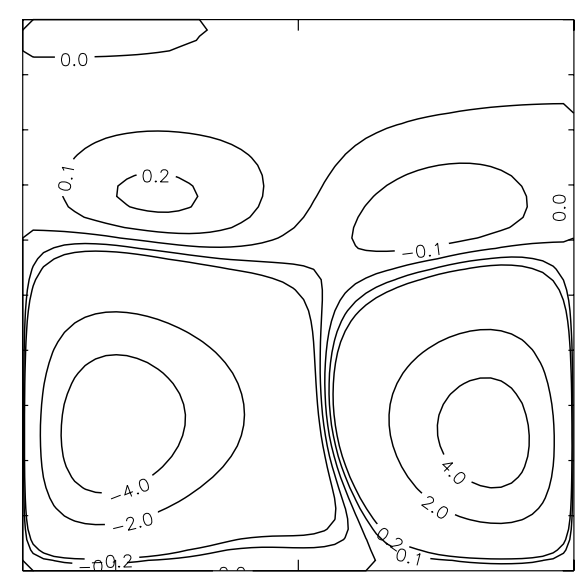

Figure 6: S Ghorai, Physics of Fluids 
(a)

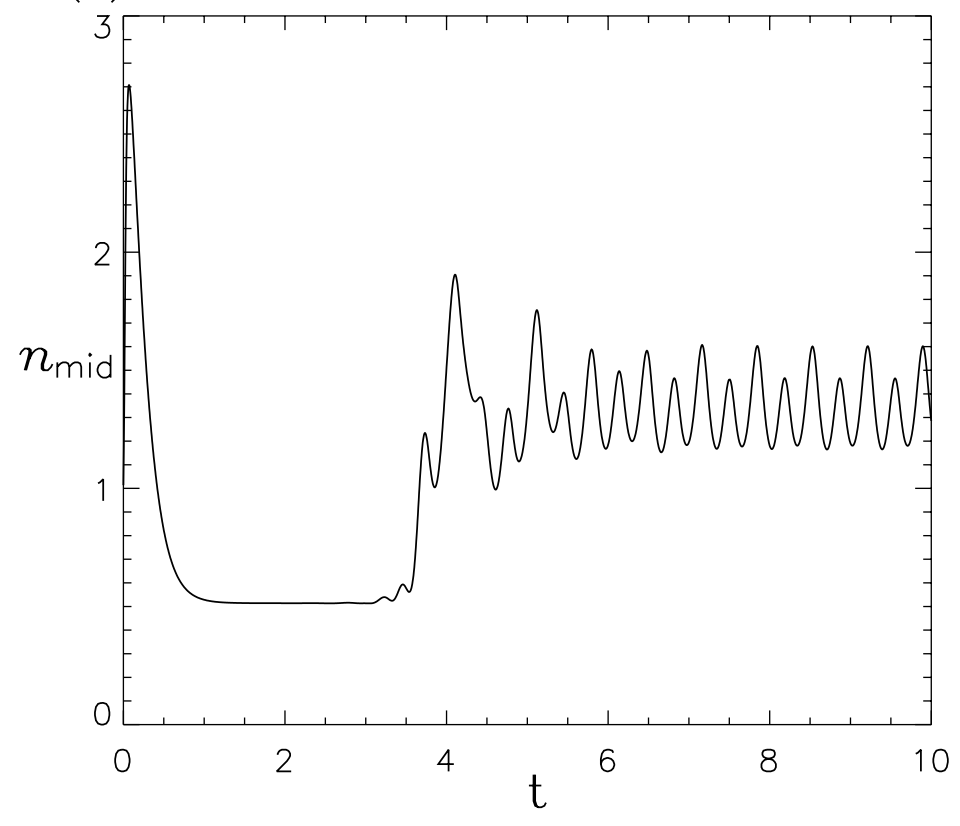

(b)

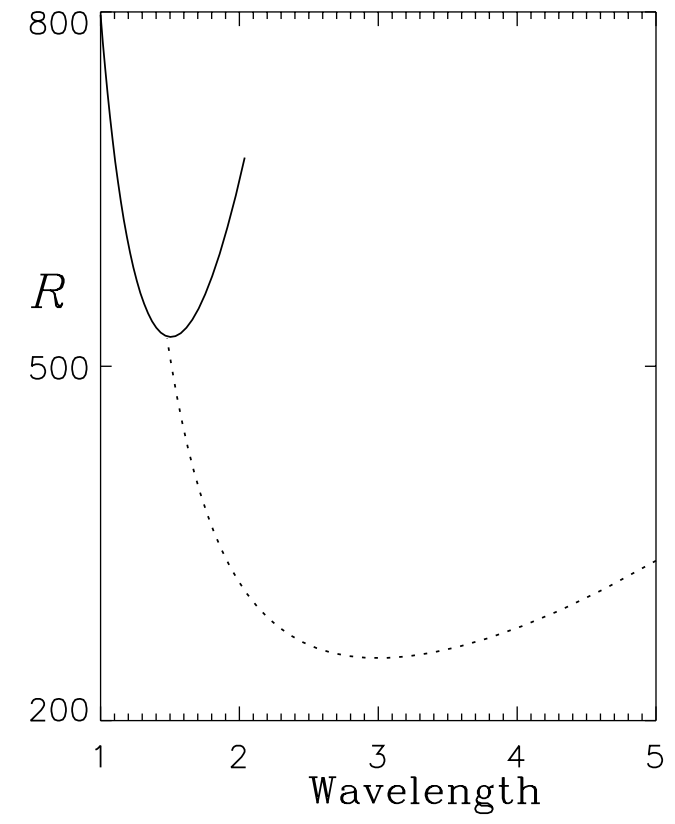

Figure 7: S Ghorai, Physics of Fluids 
(a) $t=8.40$

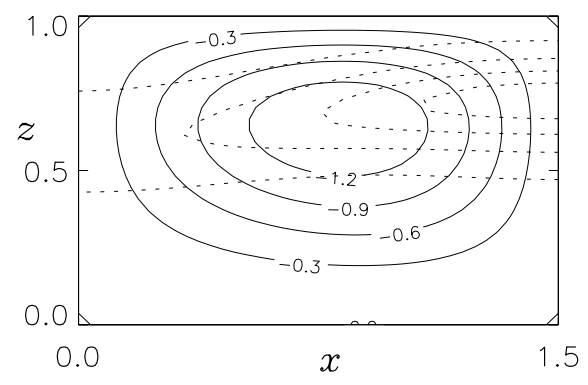

(d) $t=8.74$

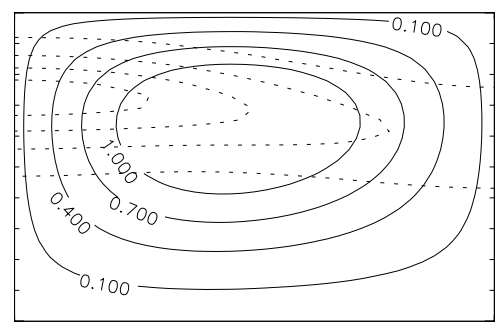

(b) $t=8.47$

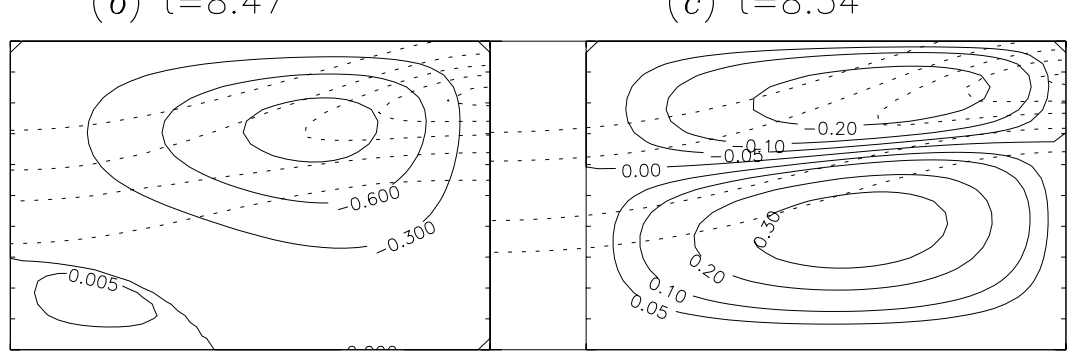

(e) $t=8.88$

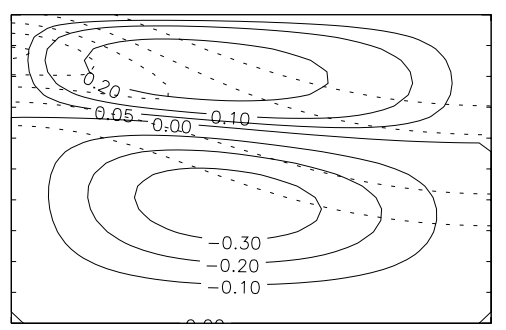

(c) $t=8.54$

(f) $\mathrm{t}=9.08$

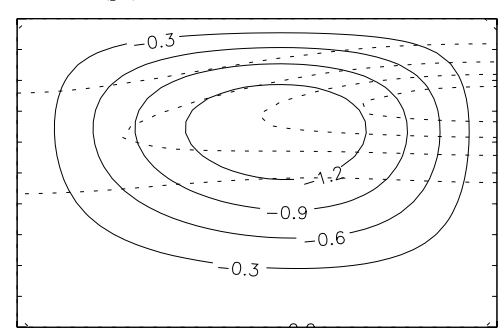

Figure 8: S Ghorai, Physics of Fluids 

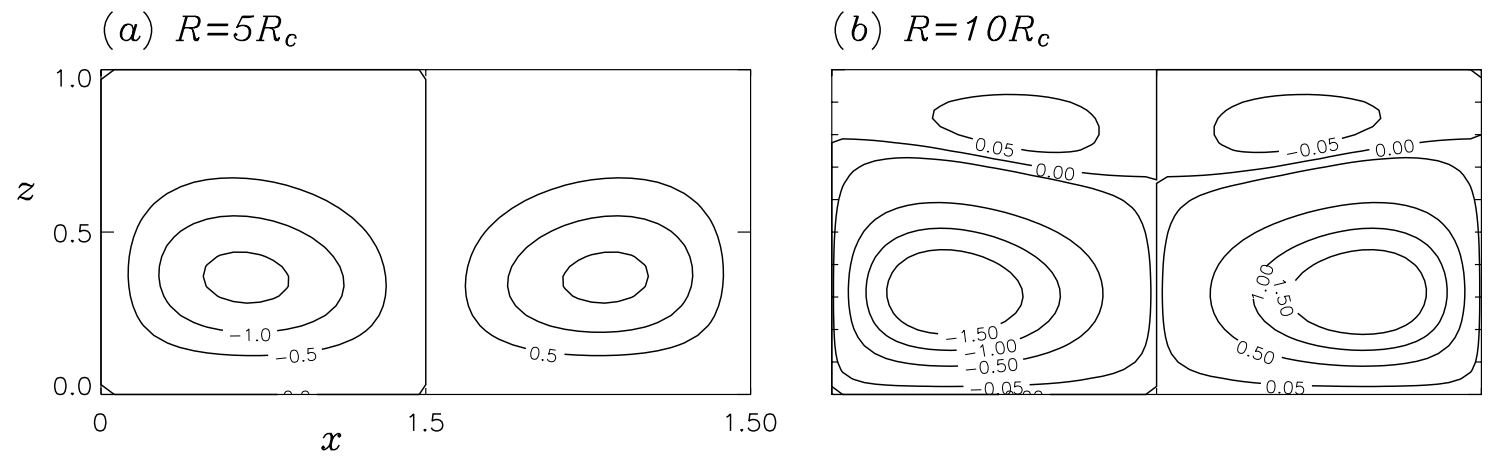

(c) $R=20 R_{c}$

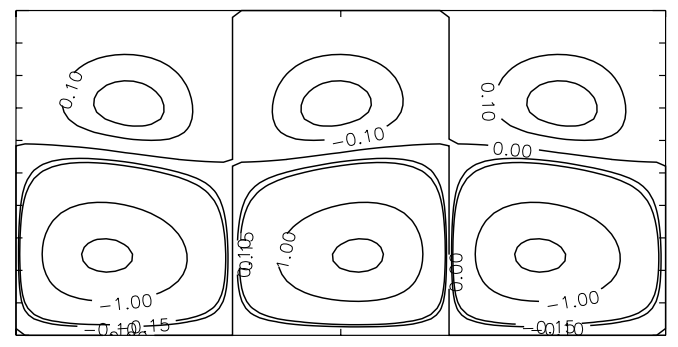

(d) $R=50 R_{c}$

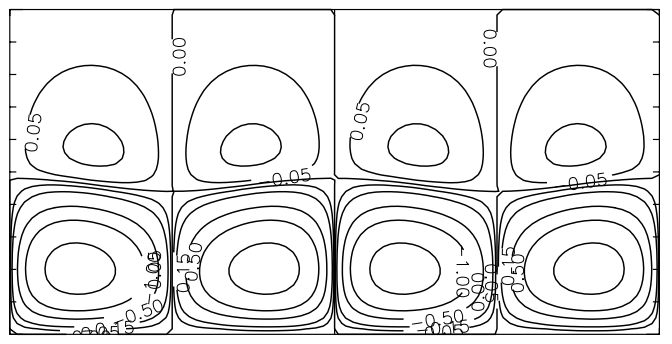

Figure 9: S Ghorai, Physics of Fluids 